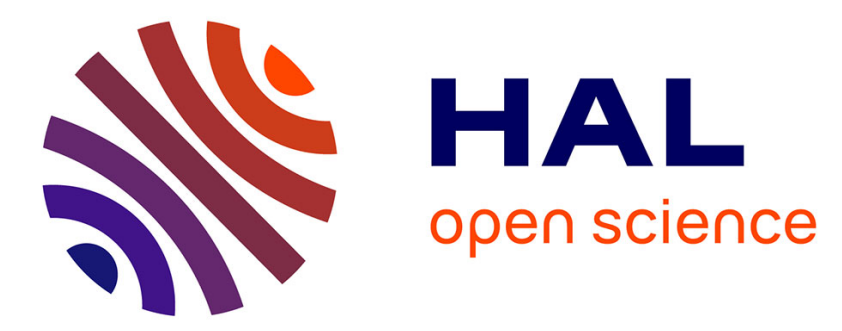

\title{
UN CHAMP MIS À L'ÉPREUVE. STRUCTURE ET PROPRIÉTÉS DU CHAMP JURIDIQUE DANS LA FRANCE CONTEMPORAINE
}

\author{
Laurent Willemez
}

\section{- To cite this version:}

Laurent Willemez. UN CHAMP MIS À L'ÉPREUVE. STRUCTURE ET PROPRIÉTÉS DU CHAMP JURIDIQUE DANS LA FRANCE CONTEMPORAINE. Droit et Société, 2015, 89, pp.129-149. halshs-01154772

\author{
HAL Id: halshs-01154772 \\ https://shs.hal.science/halshs-01154772
}

Submitted on 17 Oct 2015

HAL is a multi-disciplinary open access archive for the deposit and dissemination of scientific research documents, whether they are published or not. The documents may come from teaching and research institutions in France or abroad, or from public or private research centers.
L'archive ouverte pluridisciplinaire HAL, est destinée au dépôt et à la diffusion de documents scientifiques de niveau recherche, publiés ou non, émanant des établissements d'enseignement et de recherche français ou étrangers, des laboratoires publics ou privés. 


\title{
UN CHAMP MIS À L'ÉPREUVE. STRUCTURE ET PROPRIÉTÉS DU CHAMP JURIDIQUE DANS LA FRANCE CONTEMPORAINE
}

\author{
Laurent Willemez
}

Ed. juridiques associées | Droit et société

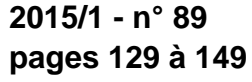

ISSN 0769-3362

ISBN 9782275028958

Article disponible en ligne à l'adresse:

http://www.cairn.info/revue-droit-et-societe-2015-1-page-129.htm

Pour citer cet article :

Willemez Laurent, « Un champ mis à l'épreuve. Structure et propriétés du champ juridique dans la France contemporaine »,

Droit et société, 2015/1 n 89, p. 129-149.

Distribution électronique Cairn.info pour Ed. juridiques associées.

(c) Ed. juridiques associées. Tous droits réservés pour tous pays.

La reproduction ou représentation de cet article, notamment par photocopie, n'est autorisée que dans les limites des conditions générales d'utilisation du site ou, le cas échéant, des conditions générales de la licence souscrite par votre établissement. Toute autre reproduction ou représentation, en tout ou partie, sous quelque forme et de quelque manière que ce soit, est interdite sauf accord préalable et écrit de l'éditeur, en dehors des cas prévus par la législation en vigueur en France. II est précisé que son stockage dans une base de données est également interdit. 


\title{
Un champ mis à l'épreuve. Structure et propriétés du champ juridique dans la France contemporaine
}

\author{
Laurent Willemez
}

Professions, Institutions, Temporalités (PRINTEMPS), Université de Versailles Saint-Quentin-en-Yvelines, 47 Boulevard Vauban, F-78047 Guyancourt cedex.

<laurent.willemez@uvsq.fr>

Résumé L'article a pour objectif de tester l'intérêt d'utiliser la notion de champ, conçue et travaillée par Pierre Bourdieu à partir de l'étude de la littérature, pour analyser les acteurs et les institutions qui font le droit et la justice dans la France contemporaine. À partir d'un retour sur de nombreux travaux sociologiques français et états-uniens, deux propriétés du champ juridique sont mises en valeur: d'une part les logiques d'autonomie, de protection des frontières mais aussi de hiérarchies internes, d'autre part la croyance commune qui relie la majorité des acteurs juridiques et judiciaires, et que l'on peut résumer par le terme de juridisme.

Pierre Bourdieu-Avocats - Langage - Profanes - Professions.

Summary A Field in Question: Structure and Properties of the Legal Field in Contemporary France

The article deals with the interest and the effectiveness of the concept of field, which was created by Pierre Bourdieu to analyze literature, in sociological research about law and justice. Thus, the article reviews French and American sociological literature about legal professionals and institutions. Two properties of the legal field are stressed: first, a logic of autonomy and gate-keeping as well as the existence of internal hierarchies; second, a common belief that is connecting most legal and judicial actors, and can be called legalism.

Pierre Bourdieu - Language - Lawyers - Laymen - Professions. 
La sociologie de Pierre Bourdieu s'est toujours présentée comme un cadre théorique général permettant au chercheur d'expliquer des phénomènes sociaux concrets et plus largement de produire une intelligibilité concernant le secteur de l'espace social qu'il étudie. Pour ce faire, le champ, l'habitus et les dispositions sont les outils théoriques privilégiés. Ils constituent des concepts omnibus qui permettent de penser l'ensemble des espaces sociaux, même s'ils ont été principalement élaborés pour expliquer les conditions de production et de réception des formes artistiques, la littérature et la peinture en particulier ${ }^{1}$, puis élargis à d'autres domaines, comme le politique et l'économique. Qu'en est-il pour le droit et la justice? Dans quelle mesure la théorie du champ offre-t-elle un cadre heuristique pour en expliquer la structuration, les modes d'organisation et les logiques propres?

Cette démarche prend d'autant plus de sens que P. Bourdieu lui-même a finalement rarement travaillé sur le terrain du droit et de la justice à proprement parler, à l'exception de quelques textes, depuis « La force du droit » ${ }^{2}$, jusqu'au chapitre sur "Les juristes, gardiens de l'hypocrisie collective» 3 , en passant par « Habitus, code et codification ${ }^{4}$ et "Droit et passe-droit ${ }^{5}$. C'est ce qui permet aux chercheurs proposant une analyse critique de ses travaux sur le droit et la justice d'affirmer que «le droit n'a jamais été une question centrale» dans "le système conceptuel du sociologue $» 6$.

Cependant, si Violaine Roussel affirme avec raison que le droit est présent «en filigrane " dans toute l'œuvre de P. Bourdieu 7 , ce n'est pas seulement parce que ses écrits montrent de quelle manière le droit constitue l'une des formes les plus achevées de domination et de violence symbolique, mais aussi parce que les acteurs et les produits du champ juridique sont bien présents dans ses recherches et dans les travaux qu'il a pu inspirer. Qu'il s'agisse du rôle joué par les juristes (en particulier les légistes) dans la genèse de l'État et de la pensée d'État, de la place du juridisme dans les rapports entre la règle et l'habitus 8 , de l'identité sociale de la profession de magistrat, ou encore des recherches sur les juristes d'affaires et l'hégémonie des juristes américains, ou enfin des deux numéros des Actes de la recherche en sciences sociales $^{9}$, tout un ensemble de travaux et de publications ont remis sur le métier

1. La récente publication des cours au Collège de France consacrés au peintre Manet constitue une bonne occasion de se replonger dans cette théorisation du champ : Pierre BouRdieu, Manet. Une révolution symbolique, Paris : Seuil-Raisons d'Agir, 2013.

2. ID., « La force du droit », Actes de la recherche en sciences sociales, 64, 1986, p. 3-19.

3. ID., «Les juristes, gardiens de l'hypocrisie collective», in François CHAZEL et Jacques Commaille (dir.), Normes juridiques et régulations sociales, Paris : LGDJ, 1993, p. 95-99.

4. Pierre Bourdieu, «Habitus, code et codification», Actes de la recherche en sciences sociales, 64, 1986, p. 40-44.

5. ID., « Droit et passe-droit », Actes de la recherche en sciences sociales, 81, 1990, p. 86-96.

6. Frédéric OCQUETEAU et Francine SOUBIRAN-PAILLET, «Champ juridique, juristes et règles de droit: une sociologie entre disqualification et paradoxe ", Droit et Société, 32, 1996, p. 10.

7. Violaine Roussel, «Le droit et ses formes. Éléments de discussion de la sociologie du droit de Pierre Bourdieu ", Droit et Société, 56-57, 2004, p. 42.

8. Rémi LenoIR, « Du droit au champ juridique », in Louis Pinto, Gisèle SAPIRO et Patrick Champagne (dir.), Pierre Bourdieu, sociologue, Paris : Fayard, 2004, p. 231-248.

9. «De quel droit?», 64, septembre 1986 ; « Droit et expertise », 76-77, mars 1989. 
l'analyse des professionnels du droit et du champ juridique. P. Bourdieu a d'ailleurs aussi conduit la réflexion sur le droit dans une autre direction, celle des acteurs et des catégories juridiques dans la construction de l'État et dans la légitimation de la domination sociale et politique 10 .

Sans méconnaître cet aspect, cet article se donne pour objectif de montrer de quelle manière le concept de champ peut constituer une hypothèse utile et heuristique pour analyser les manières dont se structurent les institutions juridiques et judiciaires et dont pensent et se pensent les professionnels du droit et de la justice. À travers la reprise de travaux de sciences sociales consacrés au droit et à la justice, il s'agit donc de comprendre en quoi la notion de champ est utile pour rendre compte de la position des juristes et de l'économie générale du droit. Et, à l'inverse, essayer de repérer dans quelle mesure l'univers du droit et de la justice permettent de mettre à l'épreuve le concept. Ce faisant, il s'agira moins de s'interroger sur les " apports » du sociologue ou les « critiques » que l'on pourrait faire à ses recherches 11 que de mettre le concept de champ à l'épreuve du terrain, conformément aux propositions méthodologiques du Métier de sociologue 12 .

$\mathrm{Du}$ fait que les concepts de P. Bourdieu sont assez rarement explicités dans toute leur étendue puisqu'ils sont sans cesse remis sur le métier et retravaillés à l'occasion de nouveaux objets de recherche, il existe diverses manières de saisir un champ. On peut cependant suivre Bernard Lahire quand il montre que le champ constitue «une formule scientifique unificatrice» permettant de penser «les pratiques au croisement des propriétés sociales des acteurs et des propriétés sociales des contextes " 13 . C'est tout particulièrement à propos du champ littéraire que les travaux de P. Bourdieu ont le plus clairement délimité le concept ${ }^{14}$. Dans ceux-ci, le champ peut être saisi de trois manières : à partir du nomos, qui en est le cœur, et des croyances qu'il produit et qui le produisent ; à partir du système de positions et de relations entre des agents, des groupes sociaux et des institutions qu'il permet d'analyser ; enfin, à partir de l'ensemble des visions du monde et des pratiques, historiquement constituées et incorporées, à partir desquelles ses agents le font vivre, le reproduisent et le transforment. Par conséquent, si l'on s'accorde sur l'idée, qu'au principe du champ, il y a le partage par les acteurs d'un certain nombre d'enjeux, mais aussi un ensemble de manières d'être et de faire définies collectivement, au travers de relations, de conflits et d'impositions hiérarchiques, alors deux questions deviennent centrales: d'une part celle de l'autonomie du

10. Pierre BourdiEu, "Esprits d’État. Genèse et structure du champ bureaucratique ", Actes de la recherche en sciences sociales, 96, 1993, p. 49-62 ; ID., Sur l'État, Paris : Seuil-Raisons d'agir, 2012.

11. Ce que proposent, d'une manière par ailleurs fort utile, plusieurs dossiers de Droit et Société, en particulier celui du numéro 32 intitulé « Norme, règle, habitus et droit chez Bourdieu » et celui du numéro 56-57, où trois articles (sur lesquels nous reviendrons) rendent un hommage plus ou moins critique à Bourdieu, mort deux ans auparavant.

12. «Le progrès théorique suppose l'intégration de données nouvelles au prix d'une mise en question critique des fondements de la théorie que les données nouvelles mettent à l'épreuve " : Pierre BouRDIEU, Jean-Claude CHAMBOREDON et Jean-Claude PASSERON, Le métier de sociologue: préalables épistémologiques, Paris : Mouton, 1973, p. 47.

13. Bernard LAHIRE, Monde pluriel : penser l'unité des sciences sociales, Paris : Seuil, 2012.

14. Pierre BouRdieu, Les règles de l'art : genèse et structure du champ littéraire, Paris : Seuil, 1991. 
champ et de l'espace des relations hiérarchiques et concurrentes qui le définissent ; d'autre part celle qui consiste à se demander ce qui est au principe du champ, cette «disposition" et ce "capital spécifique » 15 qui produisent une identité collective, celle des juristes. C'est en analysant de quelle manière ces deux propriétés ont un sens pour les institutions et les professionnels du droit et de la justice que l'on pourra mettre à l'épreuve la notion de champ juridique et discuter son extension et ses limites.

Pour ce faire, il faut s'atteler à un travail de relecture des nombreux travaux français 16 , mais aussi américains 17 , ayant étudié différentes configurations sociales dans lesquelles le champ juridique a pu jouer un rôle central, que les auteurs y aient ou non fait un usage du terme de champ et des concepts liés à une sociologie que l'on appelle «bourdieusienne»; ce faisant, il ne s'agit pas, par un acte de mauvaise foi, de faire entrer de force - quitte à les détourner - dans un cadre unique des travaux et des chercheurs, mais bien plutôt de partir de l'idée qu'un travail théorique introduit une forme de cohérence et d'homogénéité à même de produire de l'intelligibilité sur le terrain que l'on étudie. Ainsi, ce texte, qui se veut théorique, va de pair avec des enquêtes empiriques publiées depuis plusieurs années montrant l'existence d'un champ juridique ou au contraire relativisant celle-ci à d'autres moments 18 .

\section{Frontières et limites du champ juridique : autonomie, relations, hiérarchies}

Si c'est dans les Méditations pascaliennes que P. Bourdieu propose la dernière théorisation du concept de champ ${ }^{19}$, il est cependant possible d'en revenir à l'une de ses premières mises en forme, au début des années 1970, à partir d'une lecture de la sociologie des religions de Max Weber 20. Dans cet article, P. Bourdieu montre de quelle manière Weber relie la diffusion des catégories de pensée et des mythes religieux à l'émergence d'un champ religieux spécifique. En termes méthodologiques, il est donc indispensable d'étudier dans le même temps «la structure du

15. Gérard MAUGER, L'accès à la vie d'artiste : sélection et consécration artistiques, Bellecombe-en-Bauges : Éditions du Croquant, 2006.

16. Depuis plusieurs années, la sociologie, mais aussi la science politique françaises ont pris en charge l'analyse du droit et de la justice, notamment sous l'impulsion de Jacques Commaille et de la revue Droit et Société. Pour un bilan partiel de cette décennie de recherche, cf. les deux numéros de L'année sociologique intitulés « Pour une sociologie politique du droit», vol. 59, n 1 et 2, 2009.

17. Dans la mesure où les recherches en sociologie du droit et de la justice réalisées aux États-Unis proposent des analyses susceptibles de faire avancer, par une forme de comparaison implicite, les réflexions sur la situation française ; pour un exemple, cf. Jérôme PÉLISSE, «A-t-on conscience du droit ? Autour des Legal Consciousness Studies", Genèses, 2005, p. 114-130.

18. Cf. en particulier Laurent WILLEMEZ, "Quand les syndicats se saisissent du droit ", Sociétés contemporaines, 2003, p. 17-38; ID., "Engagement professionnel et fidélités militantes. Les avocats travaillistes dans la défense judiciaire des salariés ", Politix, 62, 2003, p. 145-164; ID., Des mondes ordonnés ? Professionnels du droit et militants dans la diffusion de la raison juridique, mémoire d'HDR, Versailles-Saint-Quentin-enYvelines, 2009.

19. Pierre Bourdieu, Méditations pascaliennes, Paris : Seuil, 1997.

20. ID., "Genèse et structure du champ religieux ", Revue française de sociologie, 12 (3), 1971, p. 295-334 ;

ID., "Champ intellectuel et projet créateur », Les Temps modernes, 246, 1966, p. 865-906. 
système des pratiques et des croyances religieuses » et « la division du travail religieux " 21 ; car on retrouve au cœur de ces deux phénomènes le même processus : celui de l'autonomisation du champ religieux, qui passe en particulier par la monopolisation des biens du salut par un corps de prêtres, par le fait que les profanes en soient dépossédés, ou encore par la différenciation et la hiérarchisation des activités religieuses. Cette division du travail religieux s'appuie sur les positions sociales des différents agents, et en particulier sur leur niveau de capital culturel. C'est précisément ce mouvement que P. Bourdieu analyse dans son article «La force du droit» en posant que : "La constitution du champ juridique est inséparable de l'instauration du monopole des professionnels sur la production et la commercialisation de cette catégorie particulière de produits que sont les services juridiques ${ }^{22}$. " Cette autonomie, qui est consubstantielle à la monopolisation du "droit de dire le droit ", donne aux juristes le "pouvoir de nomination », c'est-àdire la légitimité d'imposer des catégories de pensée et un langage spécifiques pour dire l'ordre social.

On comprend alors l'intérêt d'étudier la généalogie du champ juridique pour saisir de quelle manière une autorité juridique autonome a pu émerger, en lien avec l'État et avec un nouveau type de leadership politique. Quoi qu'il en soit de cette histoire, le champ juridique peut alors être considéré comme un espace social qui s'est constitué en réunissant un certain nombre d'individus, de groupes professionnels et d'institutions autour d'une sociodicée bien spécifique. Cela signifie donc qu'il existe entre ces individus, ces groupes et ces institutions des relations, des références communes, des lieux concrets ou virtuels de rencontre et d'échange. C'est enfin que ces individus et ces groupes se présentent comme des représentants du champ, défendant les intérêts du droit devenus du même coup les leurs : une de leurs activités principales est alors de définir ce que sont et ce que devraient être le droit, la justice, les professions juridiques et judiciaires ; ils s'efforcent d'en déterminer les frontières et, en interne, d'en produire les hiérarchies et les principes de division.

\section{I.1. Le champ juridique européen comme idéal-type}

Si les travaux analysant l'émergence et l'économie d'un champ juridique de ce type sont relativement rares au plan national, les réflexions sur le développement de champs juridiques transnationaux ont été assez nombreuses, qu'il s'agisse d'un champ du droit européen, d'un champ du droit international des affaires ou encore d'un champ du droit pénal international. Le retour sur certains de ces travaux permet de proposer une "formule de recherche " spécifique 23 que l'on pourrait ensuite appliquer à l'analyse du cas français. C'est en effet à partir d'enquêtes empiriques, par l'utilisation des outils de recherche particuliers que sont l'étude de trajectoires individuelles et le travail de biographie collective, la reconstitution de la

21. ID., « Genèse et structure du champ religieux », p. 306.

22. ID., « La force du droit », art. cité, p. 11.

23. Pour reprendre les termes de Jean-Michel CHAPOULIE, « La seconde fondation de la sociologie française, les États-Unis et la classe ouvrière ", Revue française de sociologie, 1991, 32 (3), p. 321-364. 
composition sociale d'un certain nombre de groupes professionnels et l'analyse des diverses positions de leurs membres, ou encore le retour sur la genèse d'institutions, que l'on peut poser l'hypothèse de l'existence d'un champ juridique, en étudier l'économie, l'extension et les limites.

C'est notamment ce que proposent un certain nombre de recherches consacrées à l'émergence du droit européen dans la seconde partie du $\mathrm{XX}^{\mathrm{e}}$ siècle, dans le sillage des travaux de Yves Dezalay. Ces chercheurs interrogent le rôle dominant du droit comme mode de légitimation des logiques transnationales, qu'il s'agisse de l'influence états-unienne en Amérique latine dans les années 1970 et $1980^{24}$, des droits de l'homme dans l'Europe de l'après-guerre ${ }^{25}$, du droit international dans les années $1920^{26}$ ou encore de la construction européenne ${ }^{27}$. Pour ce faire, ils reviennent, dans une perspective sociohistorique, sur les conditions de production de cette sociodicée particulière. À chaque fois, donc, ils reconstituent les contextes et les configurations sociopolitiques ayant permis à une des entreprises individuelles et collectives de poser, et d'imposer, souvent sur un mode conflictuel, ces différentes formes de droit (le droit des affaires, les droits de l'homme, le droit européen, le droit international) comme la manière légitime de penser et de gouverner ces espaces transnationaux. Pour ne prendre qu'un seul exemple, dans l'entredeux-guerres, le droit international émerge de la coalescence et de la cristallisation d'entreprises menées par des acteurs nationaux, en particulier des professeurs de droit - trouvant dans cet investissement une manière de «se faire " une position -, des sociétés savantes qui se consacrent au droit international et des institutions internationales comme la Société des Nations (SDN) dont les agents voient tout naturellement dans le droit international le moyen d'assurer sa position, et par conséquent la leur ${ }^{28}$. Ce sont des processus homologues, quoique évidemment tout à fait différents, que l'on retrouve dans la production du droit européen, qui est devenu aujourd'hui une véritable "métaphysique de l'Europe » 29 et l'une des seules manières légitimes de penser la construction européenne ${ }^{30}$. Pour le comprendre, il faut multiplier les enquêtes biographiques et institutionnelles, par

24. Yves DeZALAY et Bryant G. GARTH, La mondialisation des guerres de palais : la restructuration du pouvoir d'État en Amérique latine, entre notables du droit et « Chicago boys », Paris : Seuil, 2002.

25. Mikael RASK MADSEN, La genèse de l'Europe des droits de l'homme : enjeux juridiques et stratégies d'État, France, Grande-Bretagne et Pays scandinaves, 1945-1970, Strasbourg: Presses Universitaires de Strasbourg, 2010.

26. Guillaume SACRISTE et Antoine VAUCHEZ, «Les "bons offices" du droit international : la constitution d'une autorité non politique dans le concert diplomatique des années 1920 », Critique internationale, 26, 2005, p. 101-117.

27. C'était l'objet des travaux de l'équipe Polilexes (Politics of International Legal Expertise in European Societies), financés par un contrat avec l'Agence nationale de la recherche (ANR) au Centre européen de sociologie et de science politique de l'Université (CESSP) et au Centre universitaire de recherches administratives et politiques de Picardie (CURAPP).

28. Guillaume SACRISTE et Antoine VAUCHEZ, « Les "bons offices" du droit international », art. cité.

29. Antoine VAUCHEZ, «The Force of a Weak Field: Law and Lawyers in The Government of The European Union (For A Renewed Research Agenda) ", International Political Sociology, 2008, 2 (2), p. 134.

30. Qu'il faudrait sans doute discuter pour la période actuelle, le discours juridique étant sans doute en concurrence avec un discours économique et un discours gestionnaire : sur ce plan, cf. Frédéric LEBARON, La crise de la croyance économique, Bellecombe-en-Bauges : Éditions du Croquant, 2010. 
exemple sur les juristes, souvent issus du champ académique, qui ont contribué à la production des institutions européennes dans les années 1950 31, mais aussi sur les premiers juges de la Cour de justice des Communautés européennes (CJCE), ou encore, pour la même période, sur les fonctionnaires des services juridiques des institutions communautaires comme sur les responsables des services juridiques des grandes firmes multinationales et européennes ${ }^{32}$.

La reconstitution sociohistorique de ces différents champs juridiques transnationaux conduit alors à proposer une analyse de l'économie de ceux-ci. Là encore, parce qu'elle a été beaucoup étudiée sur ce plan, l'Europe offre un exemple particulièrement intéressant. Une telle recherche permet d'abord de distinguer plusieurs types de juristes, qui produisent le droit européen tout en défendant des intérêts professionnels et en circulant entre les différentes institutions et professions. D'une manière générale, on peut distinguer quatre catégories d'agents 33 : les universitaires, les juristes de la Commission européenne, les juges de la Cour de justice européenne et les avocats, consultants et autres juristes représentants d'intérêts divers. Ces différents groupes, dont il faudrait reconstituer les dispositions, les logiques pratiques, les oppositions et les hiérarchies, se retrouvent dans des espaces de production du droit (audiences judiciaires, mais aussi comités de rédaction des revues par exemple) aussi bien que dans les lieux de sociabilité bruxellois, luxembourgeois ou strasbourgeois : ce qui se joue dans ces espaces, c'est la définition d'une certaine manière de faire du droit, mais aussi la production des frontières et la mesure du montant des droits d'entrée. Toutes ces institutions font du droit un univers social autonome, en même temps qu'elles produisent et objectivent l'Europe et la construction européenne. Qui plus est, les différents acteurs circulent très volontiers entre les diverses institutions et multiplient les positions simultanées. C'est donc bien ainsi que se constitue un capital juridique européen, qui existe par-delà les enjeux professionnels opposés et les stratégies de distinction : ceux-ci n'en sont pas pour autant neutralisés, mais ils participent bien au contraire à l'organisation du champ, divisant ceux qu'unit la croyance dans le droit et dans l'Europe.

Dans ces conditions, le champ juridique européen constitue une sorte de caslimite pour saisir l'utilité de penser en termes de champ. D’abord parce que la spécificité de ces espaces transnationaux, et en premier lieu de l'Union européenne, est certes sa faiblesse constitutive, qui laisse sans doute une plus grande place à ces processus de circulation d'un groupe professionnel à un autre, d'un espace social à un autre. Comme l'explique Antoine Vauchez, c'est précisément parce que la multipositionnalité des agents du champ juridique est possible et même souhaitable que le droit constitue un espace de médiation privilégié entre des secteurs spécifiques, et qu'ainsi il détient ce magistère. En second lieu, l'intérêt heuristique du

31. Antonin COHEN, "La Constitution européenne. Ordre politique, utopie juridique et guerre froide», Critique internationale, 26, 2005, p. 119-131.

32. Antoine VAUCHEZ, « Une élite d'intermédiaires », Actes de la recherche en sciences sociales, 166-167, 2007, p. 54-65.

33. Harm SCHePel et Rein Wesseling, "The Legal Community: Judges, Lawyers, Officials and Clerks in The Writing of Europe », European Law Journal, 1997, 3 (2), p. 165-188. 
champ juridique européen tient dans le fait que la logique européenne gomme l'existence d'un certain nombre d'institutions et de logiques proprement nationales qui, dans les différents pays, retardent le développement d'un champ juridique dans toute son étendue. Les formes de défense professionnelles sont contrariées et unifient des groupes habituellement séparés, de même que les structures de socialisation à l'Europe et de circulation entre les élites conduisent les individus à aller au-delà de leurs "habitus nationaux" et de leurs identités professionnelles pour défendre une idée collective de la construction européenne et finalement des intérêts communs à l'Europe - et dans laquelle le droit joue un rôle majeur ${ }^{34}$. Dans ces conditions, l'intérêt du champ juridique européen est qu'il constitue un "idéaltype " permettant de poser les conditions de possibilité d'un champ juridique ${ }^{35}$. Et que ses propriétés, qui s'y retrouvent à l'état pur, sont beaucoup plus disséminées et parfois discrètes, voire absentes - dans l'espace français. Cela ne remet pas en cause l'intérêt de penser le champ, mais comme un concept à partir duquel on peut ordonner, à une échelle mésosociologique, une réalité sociale spécifique ${ }^{36}$.

\section{I.2. Le fait professionnel : entre effets de champ et effets de corps}

Concernant le cas français, le point central tient sans doute dans l'existence de professions juridiques et judiciaires organisées et défendant un intérêt de corps qui s'oppose apparemment à la logique du champ. Or, même si les recherches en termes de sociologie des professions n'utilisent que rarement la théorie des champs, il est pertinent d'articuler ces deux cadres. Le champ juridique s'appuie sur une série de processus liés à l'activité des représentants des groupes professionnels, et en particulier la recherche d'autonomie, d'auto-gouvernement, de production et de renforcement des frontières et de revendications territoriales, questions qui sont au cœur de la sociologie des groupes professionnels ${ }^{37}$. Comme P. Bourdieu le montre, il faut prendre au sérieux les "effets de corps" et les combiner avec les "effets de champ » 38 , en étudiant de quelle manière les représentants des professions juridiques et judiciaires, qu'il s'agisse d'ordres professionnels, d'associations ou de syndicats, réalisent un travail important de structuration, à la fois en externe et en interne. Si les « effets de champ » et les effets du groupe professionnel peuvent parfois s'opposer, ils peuvent aussi faire sens ensemble, à la fois pour contribuer à

34. Sur cette question, cf. en particulier Hélène Michel et Cécile RoBeRT (dir.), La fabrique des Européens: processus de socialisation et construction européenne, Strasbourg : Presses universitaires de Strasbourg, 2010.

35. On peut ici rappeler la définition que M. Weber donne de l'idéal-type : « On obtient un idéal-type en accentuant unilatéralement un ou plusieurs points de vue et en enchaînant une multitude de phénomènes donnés isolément, diffus ou discrets, que l'on trouve tantôt en grand nombre, tantôt en petit nombre et par endroits pas du tout, qu'on ordonne selon les précédents points de vue choisis unilatéralement, pour former un tableau de pensée homogène. " (Max WeBER, Essais sur la théorie de la science, Paris : Presses Pocket, 1995, p. 172-173.

36. Neil FuIGSTEIn et Doug McAdAM, A Theory of Fields, New York : Oxford University Press, 2012.

37. Didier DEMAZIÈRE et Charles GADEA (dir.), Sociologie des groupes professionnels: acquis récents et nouveaux défis, Paris: La Découverte, 2009; Valérie BousSARD, Didier Demazière et Philip Milburn (dir.), L'injonction au professionnalisme: analyses d'une dynamique plurielle, Rennes: Presses Universitaires de Rennes, 2010 ; Florent CHAMPY, La sociologie des professions, Paris : PUF, 2009.

38. Pierre BouRdiEU, «Effet de champ et effet de corps », Actes de la recherche en sciences sociales, 59, 1985, p. 73. 
l'autonomisation d'un champ et pour être au principe des formes de concurrence en son sein. Dans ces conditions, on pourrait dire que le champ est notamment structuré par les luttes de territoires entre groupes professionnels, et qu'il est en partie synonyme du "système des professions » que théorise Andrew Abbott lorsqu'il analyse les différents types de relations entre les groupes professionnels au sein d'un même "système ", au sein d'une " écologie » ou d' "écologies liées " : relations de domination, de division du travail, ou encore de compétition ${ }^{39}$. L'analyse du champ juridique doit alors passer par l'enquête sur l'activité de ces institutions qui produisent en même temps l'unité du champ, ses frontières et ses luttes internes.

Une partie des travaux de sociologie des professions ont analysé le travail de monopolisation d'un domaine de savoirs et d'actions au profit de ses membres. En cela, toutes les professions juridiques luttent pour que le droit soit laissé aux juristes. Il s'agit de défendre les agents du champ juridique contre des individus et des groupes prétendant aux activités juridiques (par exemple les «professions du chiffre» comme les comptables) ou, quand il n'est plus possible de fermer les frontières, de tenter de maîtriser et de réguler l'entrée de nouveaux groupes : c'est en particulier l'attitude des différents groupes d'intérêt représentant certaines professions juridiques (avocats, notaires...) face aux « réseaux interdisciplinaires » réunissant le juridique, l'audit et le consulting, que la Commission européenne préconise face aux dynamiques professionnelles des avocats qu'elle qualifie de tendances au monopole, de cartels ou d'accords collusifs et finalement d'entraves à la concurrence ${ }^{40}$. On pourrait analyser de la même manière l'unité, certes provisoire et circonstancielle, de l'ensemble des syndicats et des différents acteurs de la justice face aux projets de réforme de l'ordre judiciaire ces dernières années, ou plus encore l'action des procureurs dans les nouvelles politiques pénales : seuls représentants de l'institution judiciaire dans des instances partenariales, comme les contrats locaux de sécurité et de prévention de la délinquance, les procureurs y défendent le plus souvent les principes du droit et les valeurs judiciaires ; ce faisant, ils se positionnent d'abord comme des agents du champ juridique, alors même que toute une partie de leur activité les pousse vers l'intégration de logiques exogènes 41 .

Mais les dynamiques professionnelles jouent aussi un rôle important au cœur du champ juridique lui-même, en renforçant les concurrences et en établissant les

39. Andrew Аввотт, The System of Professions: An Essay on The Division of Expert Labor, Chicago : University of Chicago Press, 1988 ; ID., "Linked Ecologies: States and Universities as Environments for Professions ", Sociological Theory, 23 (3), 2005, p. 245-274. Il faudrait mieux distinguer le concept d'écologie et celui de champ, en montrant qu'une écologie est à la fois plus et moins qu'un champ : plus dans la mesure où elle ne se structure pas autour d'un intérêt commun mais le déborde en constituant des espaces d'action plus larges ; moins dans la mesure où elle restreint son utilité heuristique à des domaines précis sans prendre en compte les logiques de collaboration et/ou de concurrence intersectorielles. L'analyse des avocats en est un parfait exemple: si l'on suit A. Abbott, les avocats et leurs clients appartiennent à une même écologie, renvoyant à ce que l'on pourrait appeler un domaine du droit (cf. The System of Professions, op. cit., p. 122124) ; ce qui ne contredit pas l'existence d'un champ juridique mais se situe à un autre niveau d'analyse.

40. Olivier FAVEREAU (dir.), Les avocats, entre ordre professionnel et ordre marchand, Paris : Lextenso éditions, 2010.

41. Philip Milburn, Katia KostUlSKI et Denis SALAS, Les procureurs : entre vocation judiciaire et fonctions politiques, Paris : PUF, coll. « Droit et justice», 2010, p. 72-102. 
hiérarchies entre les différents professionnels. Le travail des notaires face à la réforme des professions juridiques de 1990 en fournit un exemple parmi d'autres : inquiètes d'un risque de fragilisation face à une profession d'avocat unifiée et détenant un périmètre d'action plus important, les instances de représentation des notaires luttent - avec succès - pour une égalité de traitement avec la nouvelle profession d'avocat (en particulier la capacité de salarier des notaires et la possibilité pour les conseils juridiques de devenir notaires, et pas seulement avocats) 42 .

\section{I.3. Principes de hiérarchie et processus d'unification}

L'appartenance professionnelle produit donc des divisions au sein du champ, divisions qui donnent lieu à des concurrences, à des luttes et à des hiérarchisations. $\mathrm{Au}$ sein du champ juridique, ces hiérarchies s'organisent tout particulièrement, comme P. Bourdieu l'avait montré 43 , dans l'opposition entre droit pur et droit pratique, autrement dit entre, d'une part, les théoriciens, " gardiens du temple » qui écrivent la doctrine et interprètent la législation, et, d'autre part, les praticiens qui l'appliquent au jour le jour, dans leur activité juridique et judiciaire d'écriture d'arrêts, de rédaction de contrats, de plaidoirie, ou encore l'établissement d'actes notariés. Les premiers sont principalement des universitaires, mais aussi des hauts magistrats (de la Cour de cassation ou du Conseil d'État) dont la charge est principalement de sortir les jugements de la gangue du réel pour en faire des catégories juridiques épurées; les seconds sont tous les autres professionnels qui utilisent le droit dans leur activité quotidienne. Effet de la division du travail juridique, cet antagonisme transcende ces seules professions et est fondé sur la détention de formes différentes de capital juridique.

Un second principe de division renvoie aux différents domaines du droit et aux juridictions spécifiques. Même si, comme l'écrivait Lucien Karpik en 1995, la profession d'avocat " maintient la forme dominante de la pratique généraliste » 44 , et si la carrière de magistrat du siège nécessite de passer par un ensemble d'étapes renvoyant à différentes juridictions spécialisées, il reste que ces spécialisations jouent un rôle très important dans la constitution du champ. On peut ainsi mettre en relief l'existence de sous-champs juridiques hiérarchisés, qui sont autant d'écologies spécifiques pour reprendre les termes d'A. Abbott, et dans lesquels se rencontrent les différents agents du champ mais aussi des justiciables, tous individus qui sont réunis par le domaine du droit spécifique où s'organise la rencontre entre une offre et une demande de services juridiques spécialisés 45 . En premier lieu, l'opposition entre droit public et droit privé, présentée comme la summa divisio, joue un rôle

42. Alain BERNARD, «Les notaires et la réforme du statut des professions du droit », Genèses, 27, 1997, p. 69-87.

43. Pierre Bourdieu, «La force du droit », art. cité, p. 6.

44. Lucien KARPIK, Les avocats : entre l'État, le public et le marché, Paris : Gallimard, 1995, p. 318.

45. Dans un ouvrage sur les réformes de la justice, nous avions utilisé avec Antoine Vauchez le concept de «monde du droit » pour définir non seulement des juridictions, mais aussi les réseaux de coopération et les " groupes de renfort » qui y sont actifs. Il faut cependant sans doute élargir cette notion au-delà des arènes juridictionnelles, dans l'ensemble du champ juridique : cf. Antoine VAUCHEZ et Laurent WILLEMEZ, La justice face à ses réformateurs, 1980-2006, Paris : PUF, 2007. 
central, au moins à l'Université. Au-delà, le droit apparaît comme de plus en plus éclaté, selon les compétences des juridictions, la multiplication des codes, la complexification des catégories et par conséquent les intérêts des individus, en particuliers les universitaires, à promouvoir l'autonomie de leur spécialité ${ }^{46}$. L'exemple des avocats est intéressant sur ce point, dans la mesure où les domaines du droit sont aussi hiérarchisés. S'efforçant de classer les domaines du droit, Lucien Karpik montre ainsi qu'il existe trois «familles » d'activités, liées à trois « savoirs » : le « savoir des affaires » (regroupant le droit de la fiscalité, de la propriété intellectuelle, de l'international, des sociétés, des transports et du pénal financier) ; le « savoir intermédiaire" (regroupant le droit commercial, des professions et des transports) et le " savoir des particuliers » (qui inclut le droit pénal, le droit des personnes, des assurances, des loyers et du travail) ${ }^{47}$. D'une manière proche, les sociologues du droit états-uniens ont souligné les différentes formes de stratification de la profession en mettant l'accent sur l'existence de deux « hémisphères » de la profession de lawyer: l'une est tournée vers des clientèles personnelles, l'autre vers une clientèle d'entreprises ; cette différence renvoyant à de fortes différences de classe, de genre et de race ${ }^{48}$. Ils montrent alors la hiérarchisation sociale très prégnante au sein de la profession, depuis les avocats vivant de l'aide juridictionnelle, et par conséquent faisant l'expérience de la marginalité ${ }^{49}$, jusqu'à l'élite du barreau, elle-même particulièrement stratifiée ${ }^{50}$. Même si ces travaux ne peuvent être importés sans précaution dans la société française ${ }^{51}$, les méthodes utilisées et les logiques d'analyse pourraient et devraient être largement transférées sur les professions juridiques et judiciaires françaises.

Mais ces logiques de division sont concurrencées par des processus d'unification du champ juridique, qui se jouent dans un certain nombre d'institutions et d'arènes. Il ne faut tout d'abord pas oublier les va-et-vient entre différents lieux juridiques et les multipositionnalités d'un certain nombre d'individus : professeurs de droit présents dans les cabinets ministériels (donc dans un travail de rédaction des lois), ou dans des activités de consultation, voire de plaidoirie ou d'arbitrage ${ }^{52}$, avocats ou magistrats chargés de cours ou professeurs associés dans les universités, hauts fonctionnaires devenant avocats, etc. Circulant d'une arène à une autre, multipliant les positions successivement ou simultanément, ces agents se sont constitués lors de leurs études

46. François GRUA, « Les divisions du droit », Revue trimestrielle de droit civil, 1993, p. 59.

47. Lucien KARPIK, « Avocat : une nouvelle profession?», Revue française de sociologie, 26 (4), 1985, p. 571-600.

48. John HeINZ et Robert Nelson, Urban Lawyers: The New Social Structure of the Bar, Chicago : University of Chicago Press, 2005.

49. Marina ZALOZNAYA et Laura Beth NIELSEN, « Mechanisms and Consequences of Professional Marginality: The Case of Poverty Lawyers Revisited », Law \& Social Inquiry, 36 (4), 2011, p. 919-944.

50. Ronit DinOvitzer, "The Financial Rewards of Elite Status in the Legal Profession », Law \& Social Inquiry, 36 (4), 2011, p. 971-998.

51. En particulier du fait de la différence entre les lawyers et les avocats: sur cette question, voir Anne Boigeol, "The French Bar: The Difficulties of Unifying a Divided Profession ", in Richard ABEL et Philip WILlis (eds.), Lawyers in Society, vol. 2: The Civil Law World, Chicago : University of Chicago Press, 1988, p. 258-294.

52. Géraud de Geouffre de la Pradelle, "Les professeurs de droit », in Daniel Soulez Larivière et Hubert DALLE (dir.), Notre justice, Paris : Robert Laffont, 2002, p. 160-171. 
communes dans les facultés de droit ou dans les Instituts d'études politiques un capital juridique et un capital social qui sont à la fois propres à leur activité professionnelle spécifique et communs à l'ensemble de leurs engagements. La conception du droit que la plupart de ces juristes défendent est construite historiquement dans les facultés de droit autour d'un modèle civiliste et légicentrique ${ }^{53}$. Ce dernier nourrit aujourd'hui encore les visions du monde d'un certain nombre de juges de la Cour suprême et de professeurs de droit, qui pratiquent ce que l'un d'entre eux appelle le "fétichisme du texte de loi ", rejetant comme fondamentalement non valable toute forme d'interprétation et refusant l'intégration de la jurisprudence. Cette vision quasi aristocratique de l'activité juridique est ce qui a longtemps distingué le «droit continental» du droit anglo-saxon 54 .

En France, l'unification du champ juridique, et par conséquent l'affaiblissement des effets de corps ont été renforcés depuis le début des années 1980 et accentués depuis les années 2000 par les transformations profondes de l'activité juridique: Yves Dezalay montre dans quelle mesure "l'aggiornamento dans le champ du droit " a remis en cause la division traditionnelle du travail de domination symbolique ${ }^{55}$. Il explique de quelle manière «la revalorisation savante du droit des affaires ou du droit commercial en droit économique [...] apparait comme le corollaire d'une reconnaissance par l'ensemble du corps du poids et du nouveau statut des praticiens intéressés par ce marché où leur place dépend du label de juridicité accordé à la redéfinition des pratiques et des représentations juridiques légitimes qu'ils revendiquent comme adéquats à ces nouveaux enjeux sociaux » 56 . Autrement dit, non seulement les proximités s'accroissent entre les différents groupes professionnels liés au droit des affaires, mais en outre on assiste à des transferts de légitimité d'une institution à l'autre : comme les autres établissements d'enseignement supérieur dans de nombreux espaces sociaux, la faculté de droit - mais aussi la publication d'articles de doctrine - apparaît de plus en plus, pour des praticiens, comme un lieu parmi d'autres d'obtention de nouvelles ressources, en l'occurrence de capital symbolique monnayable sur les différents marchés juridiques. À l'inverse, les universitaires y trouvent des rétributions - symboliques mais aussi matérielles - de même que des possibilités de débouchés pour leurs étudiants, et donc un meilleur positionnement de leur faculté de droit (ou de leur master) sur ce qui est devenu le marché de l'enseignement supérieur. Autant dire que parler d'un champ ne signifie précisément pas que l'on postule l'unité et l'identité des intérêts, mais bien une forme de dialectique entre d'une part des intérêts divers et divergents, et d'autre part le partage d'un certain nombre de valeurs et de croyances.

53. Guillaume SACRISTE, La République des constitutionnalistes : professeurs de droit et légitimation de l'État en France, 1870-1914, Paris : Presses de Sciences Po, 2011, notamment p. 46-52.

54. "Civil law academicians [...] have been concerned almost exclusively with the conceptual elaboration and formal rationalization of doctrine, processes requiring little sensitivity to the factual nuances of concrete cases or to the societal interests they implicate», Mark J. OsIEL, «Lawyers as Monopolists, Artistocrats, and Entrepreneurs ", Harvard Law Review, 103 (8), 1990, p. 2052.

55. Yves DeZalay, «Juristes purs et marchands de droit. Division du travail de domination symbolique et aggiornamento dans le champ du droit ", Politix, 10-11, 1990, p. 70-91.

56. ID., «Le droit des faillites : du notable à l'expert », Actes de la recherche en sciences sociales, 76, 1989, p. 27. 
Cette double réalité de la concurrence et de l'unification se manifeste particulièrement clairement dans les luttes mettant aux prises les institutions de socialisation et de formation au droit qu'analysent les chercheurs réunis dans la recherche collective Elidroit 57 . Les auteurs montrent bien les formes de concurrence de plus en plus sévères entre facultés, grandes écoles, voire écoles professionnelles pour la définition d'un «bon » enseignement du droit et du «bon juriste ». Ils mettent aussi l'accent sur les spécificités de l'enseignement en droit selon les espaces professionnels concernés. C'est précisément ainsi que le concept de champ peut être repris, dans les concurrences autour de l'imposition d'une définition commune de ce que sont le droit et l'activité juridique et des manières de l'acquérir.

\section{Le nomos et l'illusio : le juridisme}

Il devient alors nécessaire de revenir sur ce qui réunit la plupart des agents du champ juridique, et que l'on pourrait résumer par une disposition commune, plus ou moins forte mais toujours présente : le juridisme. Cette disposition, apportée par une socialisation commune (via les formations et l'expérience professionnelle), dessine pour chaque individu un univers de façons de faire son métier mais aussi de voir le monde, et au final des catégories de pensée et un «sens pratique professionnel» communs ou du moins proches 58 . En sachant que c'est bien entendu la combinaison d'une position des individus dans l'espace social (à travers les capitaux qu'ils détiennent) et de la place occupée par le groupe professionnel dans un champ particulier qui permet de comprendre les manières spécifiques et différenciées de faire et concevoir son métier ${ }^{59}$, on peut étudier dans quelle mesure le champ juridique produit des façons de penser et de faire communes à l'ensemble de ceux qui agissent en son sein.

Cette disposition, centre géométrique du champ, produit d'abord la croyance que tous les acteurs de ce champ juridique partagent : celle de la primauté du droit comme mode de régulation et comme valeur suprême permettant le bon gouvernement des sociétés. C'est dire qu'au-delà des divisions et des hiérarchies, le champ est fondé sur des croyances communes, une illusio, que partagent tous les acteurs impliqués dans le champ ; comme l'écrit P. Bourdieu dans Les méditations pascaliennes, « la disposition constituante [...] est cette adhésion tacite au nomos, cette forme particulière de croyance, l'illusio, qu'exigent les champs scolastiques et que suppose la mise en suspens des objectifs de l'existence ordinaire, au profit d'enjeux nouveaux, posés et produits par le jeu même» 60 . Si ce nomos peut exister

57. Dirigée par Liora Israël et Rachel Vanneuville : cf. en particulier le dossier dans le numéro 83 de Droit et société (2013) intitulé "Les enjeux contemporains de la formation juridique»; le projet est notamment résumé dans l'introduction du dossier : Myriam AïT-AOUdiA et Rachel VANNEUVILLE, «Le droit saisi par son enseignement ", p. 7-16.

58. Romuald BoDin, « Une éducation sentimentale », Déviance et Société, 35 (1), 2011, p. 93-112.

59. Pour une analyse des relations entre positions sociales des magistrats, entrée dans la profession et rapport au métier, cf. Alain BANCAUD, La haute magistrature judiciaire entre politique et sacerdoce ou Le culte des vertus moyennes, Paris: LGDJ, 1993 ; Pierre CAM, "Juges rouges et droit du travail», Actes de la recherche en sciences sociales, 19, 1978, p. 2-27.

60. Pierre Bourdieu, Méditations pascaliennes, op. cit., p. 122. 
chez les agents à l'état non conscient, c'est parce qu'il est incorporé sous la forme de manières d'être, de manières de faire, de formes de pensée et de classification.

Les articles de P. Bourdieu sur le champ juridique, appuyés sur des travaux empiriques de doctorants ou de collègues présentés dans les séminaires qu'il a animés, permettent de synthétiser quelques éléments de cette "disposition constituante», productrice au final d'un «habitus juridique». D'abord, montrant que le droit exerce un effet non seulement sur les justiciables, mais aussi «sur ceux qui exercent cet effet ", il insiste sur ce qui réunit les juristes : c'est de bonne foi que ceuxci croient à l'efficacité du droit comme contribuant à la pacification et à la rationalisation du monde, qu'ils le pensent "pur et parfaitement autonome » et qu'ils dénient l'existence de logiques exogènes dans l'exercice d'activités juridiques ${ }^{61}$. C'est ensuite dans un langage spécifique que P. Bourdieu voit une des caractéristiques propres au champ juridique: fondé sur un triple effet d'apriorisation, de neutralisation et d'universalisation, il nécessite dès lors, pour ceux qui prétendent l'utiliser, la détention de ressources et de compétences spécifiques, que P. Bourdieu résume sous l'expression quelque peu tautologique de «sens juridique» ou d'«esprit juridique»62. Enfin, l'ensemble de ces éléments produit un ethos particulier, marqué par une ascèse, une réserve et une distance quasi aristocratique dans la mesure où elle fait penser à un sens de l'honneur traditionnel. Au total, cette "disposition constituante " pourrait être résumée sous le terme de "juridisme», dans le sens où, comme l'écrit Rémi Lenoir, «le juriste tend à enregistrer les pratiques sociales en leur donnant les apparences de l'évidence logique » 63 ; si le juridisme n'appartient pas qu'aux juristes 64 et constitue un « sous-produit » de la disposition scolastique, il reste que cet "esprit juridique» se retrouve presque à l'état pur chez nombre d'agents du champ juridique. Son effet principal est de distinguer les profanes des professionnels du droit, dont la légitimité est alors constituée par une forme de sacré, traduite par ce souci particulier pour la forme et par cette propension à l'auto-référence des pratiques et des discours.

\section{II.1. Le formalisme et la distance}

L'élément premier et central de la disposition juridique renvoie à une préoccupation forte et presque exclusive pour la forme et le formalisme : que ce soit à partir de la "pureté » de constructions théoriques doctrinales réalisées par les professeurs de droit 65 , de la technicité hors-pair d'un contrat rédigé par un avocat, ou encore

61. ID., «Les juristes, gardiens de l'hypocrisie collective», art. cité, p. 96-99. Bien entendu, tous les juristes n'adhèrent pas en bloc et de manière homogène à cette croyance. Il faudrait montrer dans quels contextes sociopolitiques et à partir de quelles dispositions individuelles et de quelles socialisations professionnelles sont susceptibles de se développer des logiques de résistance au sein même du champ, des déviances et des tentatives de subversion de l'ordre juridique. Pour une réflexion globale sur ces questions, cf. Jacques LAGROYE, Appartenir à une institution. Catholiques en France aujourd'hui, Paris : Economica, 2009.

62. Pierre Bourdieu, « La force du droit », art. cité, p. 5.

63. Rémi LENOIR, «Du droit au champ juridique », art. cité.

64. Pierre Bourdieu, Esquisse d'une théorie de la pratique, Genève : Droz, 1972.

65. Yves DeZALAY, «La production doctrinale comme objet et terrain de luttes politiques et professionnelles », in Yves PoIRMEUR (dir.), La doctrine juridique, Paris : PUF-CURAPP, 1993, p. 230-239. 
de la précision d'un arrêt ou du respect scrupuleux de la procédure par un magistrat 66 , c'est bien d' " art juridique " qu'il s'agit, valorisant le travail juridique comme une sorte d'artisanat. L'excellence juridique renvoie à la détention d'un ensemble de ressources à la fois techniques et culturelles. Ces ressources sont acquises dans des écoles professionnelles, en France dans les facultés de droit d'abord, mais aussi dans les écoles du barreau, l'École nationale de la magistrature (ENM) ou l'École nationale des greffes (ENG), aujourd'hui dans les instituts d'études politiques ou encore les écoles de commerce ${ }^{67}$. Comme le montre Anne Boigeol, la création de l'ENM en 1958 renvoie au souci de formalisation et de codification des savoirs et des savoir-faire aux dépens de l'identité notabiliaire des anciens magistrats ${ }^{68}$. C'est sans doute chez les plus hauts magistrats que l'on retrouve à l'état presque pur cet aspect de l'habitus juridique : «Ce qui fonde leur autorité [il s'agit d'une partie des magistrats de la Cour de cassation, qu'Alain Bancaud qualifie de "magistrats dogmatiques”] c'est, outre leur conformité aux valeurs sacerdotales de la justice, leur maîtrise de la "technique juridique", et plus particulièrement de la technique de la Cour de cassation. Ils se font en particulier une spécialité de la précision des termes, de l'intransigeance grammaticale, de la rigueur du raisonnement, des tournures lapidaires 69 . "

Mais ces compétences expressives, juridiques et techniques doivent s'accompagner de ce qu'Antoine Vauchez appelle "une intelligence de la situation d'espèce ": les magistrats se doivent de faire preuve de "qualités humaines " et d'écoute, mais aussi d'inventivité face à des situations compliquées 70 . De même, les travaux américains sur les avocats, en particulier sur ceux qui sont spécialisés dans les « services juridiques personnels " (droit du divorce, droit du travail, «petit » droit pénal, etc.) montrent que ce qui est souligné, c'est la capacité d'écoute de ces avocats, les qualités humaines qui leur permettent de mieux représenter leurs clients 71 ; le travail quotidien de négociation et d'ajustement entre clients et avocats met au centre de la légitimité de la profession la «compétence relationnelle» 72 . La technicité juridique et l'excellence scolastique n'ont alors d'efficacité sociale véritable que dans la mesure où, comme dans le rapport à la « haute culture »,

66. Violaine RousSEL, «Les changements d'ethos des magistrats », in Jacques Commaille et Martine KaLUSZYNSKI (dir), La fonction politique de la justice, Paris : La Découverte, 2007, p. 25-46.

67. Émilie BILAND, «Quand les managers mettent la robe. Les grandes écoles de commerce sur le marché de la formation juridique ", Droit et Société, 83, 2013, p. 49-65.

68. Anne BoIGEOL, «La formation des magistrats : de l'apprentissage sur le tas à l'école professionnelle », Actes de la recherche en sciences sociales, 76, 1989, p. 49-64.

69. Alain BANCAUD, "Une "constance mobile" : la haute magistrature", Actes de la recherche en sciences sociales, 76,1989 , p. 41.

70. Antoine VAUCHEZ, «Le juge, l'homme et la “cage d'acier”. La rationalisation de l'activité judiciaire à l'épreuve du "moment Outreau" ", in Hélène Michel et Laurent Willemez (dir.), La justice au risque des profanes, Paris : PUF-CURAPP, 2007, p. 31-52.

71. Tanina Rostain, "Professional Power: Lawyers and The Constitution of Professional Authority", in Austin SARAT (ed.), The Blackwell Companion to Law and Society, Blackwell Publishing: Malden, 2004, p. 146-169.

72. Philip MilbuRn, «La compétence relationnelle : maîtrise de l'interaction et légitimité professionnelle. Avocats et médiateurs », Revue française de sociologie, 43 (1), 2002, p. 47-72. 
une distance peut être prise avec elles et que la règle a aussi pour effet de distinguer ceux qui sont en mesure de la transgresser ou de s'en exempter 73 .

\section{II.2. Un langage spécifique}

On comprend alors l'importance jouée par le langage spécifique au champ juridique. Celui-ci se marque d'abord par un ensemble de propriétés grammaticales et de vocables : verbes à l'indicatif valant des impératifs, mise à distance de la langue naturelle au profit de qualifications juridiques décontextualisées, utilisation d'un jargon ritualisé... La marque principale du discours juridique est son éloignement des façons usuelles de parler et d'écrire ${ }^{74}$. C'est à travers ces pratiques discursives particulières que se produit, se reproduit et s'entretient la proximité entre les différents agents du champ parlant la même langue. Comme le montre Basil Bernstein, l'existence d'un "code spécifique qui détermine les actes verbaux " d'un groupe social est à la fois le résultat de l'intériorisation des rapports sociaux au sein du groupe et l'un des éléments qui conditionnent l'identité sociale de ce groupe ${ }^{75}$. L'analyse des pratiques linguistiques, écrites comme orales, est donc l'une des conditions de la définition de la disposition juridique.

Plus précisément, comme l'a montré P. Bourdieu, ce langage juridique a pour principale caractéristique d'être un élément d'encodage et de retraduction en catégories globales et à vocation universalisante : les travaux états-uniens des années 1970 et 1980 ont mis l'accent sur le rôle du langage juridique dans la transformation des différends en litiges, puis éventuellement en procès, à travers des processus d'élargissement, de désingularisation et de montée en généralité du discours ${ }^{76}$. Si c'est de cette activité spécifique que les juristes tirent une grande partie de leur pouvoir symbolique, celle-ci est aussi un puissant facteur d'homogénéisation du champ : qu'il s'agisse d'avocats spécialisés dans le divorce faisant émerger la volonté de leurs clients quant à l'avenir de leur situation matrimoniale ${ }^{77}$, de substituts du procureur qualifiant juridiquement les infractions constatées par la police 78, ou encore de professeurs de droit réussissant par leurs consultations à transformer le droit de propriété en droit constitutionnel 79 , à chaque fois il s'agit de réaliser un travail de taxinomie, de production de catégories à partir du « codage de cas singu-

73. Pierre Bourdieu, « Droit et passe-droit», art. cité.

74. Jerzy WroblewSKI, «Les langages juridiques : une typologie », Droit et Société, 8, 1988, p. 13-27.

75. Basil BeRnSteIn, Langages et classes sociales : codes socio-linguistiques et contrôle social, Paris : Éditions de Minuit, 1975, p. 192-193.

76. Lynn MATHER et Barbara YNGVESson, « Language, Audience, and The Transformation of Disputes », Law \& Society Review, 15 (3-4), 1988, p. 775-821; Austin SARAT, Richard L. ABEL et William L. F. FelSTINER, "L'émergence et la transformation des litiges : réaliser, reprocher, réclamer », Politix, 16, 1991, p. 41-54.

77. Benoit BASTARD, Les démarieurs : enquête sur les nouvelles pratiques du divorce, Paris : La Découverte, 2002, p. 115 et suiv.

78. Angèle CHRISTIN, Comparutions immédiates : enquête sur une pratique judiciaire, Paris : La Découverte, 2008.

79. Hélène MiCHEL, «Une reconfiguration du jeu politique par le droit: défense du droit de propriété et saisine du Conseil constitutionnel ", in Liora ISRAËL, Guillaume SACRISTE, Antoine VAUCHEZ et Laurent WILLEMEZ (dir.), Sur la portée sociale du droit. Usages et légitimité du registre juridique, Paris : PUF-CURAPP, 2005, p. 171-183. 
liers ", de la même manière que le font par exemple les statisticiens ${ }^{80}$. Tout l'enjeu $\mathrm{du}$ discours juridique est alors de produire des catégories performatives, et c'est d'ailleurs leur éloignement relatif de la langue naturelle qui est, pour reprendre les termes de John L. Austin, la condition de félicité de ces énonciations ${ }^{81}$ : l'efficacité sociale du discours juridique tient à la fois au fait que les catégories produites sont perçues comme des universels (au sens où leur robustesse permet à d'autres juristes de les utiliser pour d'autres cas et dans d'autres contextes) et que, dans sa forme grammaticale et son vocabulaire, la langue utilisée produit de la distance par rapport au profane : c'est là une condition de possibilité de perpétuation du monopole des juristes.

\section{II.3. Autonomie et désintéressement}

«L'esprit juridique» se marque ensuite traditionnellement par un sentiment sinon d'indépendance, du moins d'autonomie par rapport à des réalités exogènes. Cette représentation d'une autonomie de l'action et d'une distance au politique autant qu'à l'économique est certes au fondement de la genèse du champ juridique, mais elle est incorporée par les individus eux-mêmes, au point de devenir consubstantielle à leur identité professionnelle. Dans ses travaux sur les magistrats pénalistes, et plus précisément sur les juges d'instruction, Rémi Lenoir montre ainsi comment «le magistrat tire son autorité de son insertion dans l'institution judiciaire et donc de sa conformité aux normes régissant sa fonction », occultant et déniant ce qui lui est extérieur 82 .

Mais c'est bien entendu pour les avocats que la question de l'indépendance par rapport aux logiques économiques joue le rôle le plus important, au point qu'elle constitue une véritable idéologie professionnelle, à la fois « contrainte et écran » 83 . Il faut prendre au sérieux la manière dont les codes et les instances de contrôle de la profession ont, depuis sa naissance, mis le désintéressement et l'indépendance au cœur de l'organisation du groupe, et donc de la légitimité de chacun : selon L. Karpik, cette morale professionnelle (au sens durkheimien) est le ciment du groupe en France et lui a permis pendant des siècles de préserver son autonomie, à bonne distance du marché et de l'État ${ }^{84}$. Aux États-Unis aussi, l'autonomie est un fondement historique de la profession : aujourd'hui encore, les avocats d'affaires continuent de revendiquer une autonomie par rapport à leurs clients, et ce contre toute évidence ${ }^{85}$. Insister sur l'intérêt au désintéressement des avocats ne signifie donc en rien que ceux-ci - ou en tout cas une partie d'entre eux - n'adhèrent pas à cette

\footnotetext{
80. Alain DESROSIÈRES, La politique des grands nombres : histoire de la raison statistique, Paris : La Découverte, 1993, p. 291.

81. John Langshaw Austin, Quand dire, c'est faire, Paris : Seuil, 1970.

82. Rémi LENOIR, « La parole est aux juges », Actes de la recherche en sciences sociales, 101-102, 1994, p. 79-80.

83. Anne BoIGEOL, « De l'idéologie du désintéressement chez les avocats », Sociologie du travail, 1, 1981, p. 79.

84. Lucien KARPIK, Les avocats, op. cit.

85. Robert Eli RosEN, « Rejecting the Culture of Independence: Corporate Lawyers as Committed to Their Clients ", Studies in Law, Politics and Society, special issue " Law Firms, Legal Culture, and Legal Practice ", 52, 2010, p. 33-71.
} 
valeur, ni qu'elle ne constitue pas pour eux un puissant moteur à l'activité professionnelle, bien au contraire ${ }^{86}$. De fait, au-delà de la profession d'avocat, les thèmes de l'autonomie, de l'indépendance et de la gratuité contribuent à autoriser les juristes à conserver dans les pays européens un statut social élevé, bref à se constituer un capital symbolique 87 .

\section{II.4. Dispositifs et pratiques d'exclusion des profanes}

$\mathrm{Au}$ final, l'ensemble des composantes de cette disposition juridique produit d'abord un effet de séparation entre juristes et non-juristes, entre savants et profanes. Cette distinction, incorporée par les agents, s'inscrit aussi dans nombre de pratiques sociales et de dispositifs la mettant en scène et la renforçant.

L'architecture judiciaire, souvent de style néo-classique, imposante et intimidante 88 , le costume particulier des professionnels du droit, dont les différences renvoient d'ailleurs à des codes objectivant les hiérarchies au sein du champ, enfin les rituels et toutes les cérémonies sacralisant le moment judiciaire sont autant de facteurs d'exclusion des profanes en même temps qu'ils nourrissent un sentiment de communauté et d'appartenance au champ. Ils s'inscrivent dans des rites d'interaction au sens d'Erving Goffman, permettant aux « interactants » de «s'accréditer mutuellement » et de co-produire ensemble «l'ordre expressif en vigueur» 89 . Dans son enquête ethnographique sur la justice des mineurs, Patricia Benec'h Le Roux a montré combien ces rites d'interaction structuraient les échanges des divers professionnels de la justice et écartaient ainsi de l'interaction les justiciables, qui ne comprenaient pas, par exemple, les formes de courtoisie et de déférence entre magistrats, avocats et greffiers ${ }^{90}$. Ces rituels renvoient d'ailleurs aussi à des règles déontologiques et procédurales, qui institutionnalisent les échanges entre les différents acteurs de la scène judiciaire, formalisent et objectivent une proximité de champ, et ce faisant approfondissent le sentiment d'incompréhension souvent vécu par les justiciables, en particulier par ceux qui sont les plus éloignés du droit. Retenue dans l'expression des émotions, interdiction de certaines prises de parole, modération dans l'affirmation des désaccords : cette police de l'audience est imposée aux profanes et a pour effet de souder les différents membres des groupes professionnels participant au dispositif.

Mais ces rites d'interaction s'accompagnent de pratiques à la fois plus incluantes (à l'intérieur du champ) et plus excluantes (pour l'extérieur), et que l'on peut qualifier de rites d'institution : on sait que, selon P. Bourdieu, l'intérêt du rite d'institution n'est pas dans le passage d'un état à un autre qu'il permet, mais dans le fait même qu'il

86. Frédéric LORDON, L’intérêt souverain : essai d'anthropologie économique spinoziste, Paris : La Découverte, 2006.

87. Mark J. OsIEL, «Lawyers as Monopolists, Artistocrats, and Entrepreneurs », art. cité.

88. «Le Palais de Justice doit en imposer, voire intimider, dans l'intérêt de la Nation et, dans la logique de convenance stylistique propre à l'éclectisme, seul le néo-classicisme peut être admis pour exprimer le prestige du pouvoir judiciaire et marquer dans la ville son caractère immuable», Claude LOUPIAC, «Du temple de Thémis à la Maison des droits de l'homme ", Sociétés et représentations, 12, 2001, p. 295.

89. Erving Goffman, Les rites d'interaction, Paris, Éditions de Minuit, 1988, p. 33 et 36.

90. Patricia Benech-Le Roux, Au tribunal pour enfants: l'avocat, le juge, le procureur et l'éducateur, Rennes : PUR, 2008 ; cf. aussi Liora IsRAËL, «Les mises en scène d'une justice quotidienne », Droit et Société, 42-43, 1999, p. 393-421. 
établit une frontière entre ces deux états ${ }^{91}$. Toute une série de rites rythment la vie des agents du champ juridique : soutenances de thèse, leçons d'agrégation ou rentrée solennelle pour le sous-champ académique, épreuves (écrites et orales) de concours dans les différentes écoles d'application (École française du barreau, ENM), prestations de serment des nouveaux magistrats et des nouveaux avocats, rentrées solennelles au début de chaque année judiciaire... Si ces rituels finissent parfois par ne plus s'accompagner de croyances, ils contribuent cependant à reproduire les institutions et les frontières du champ: ils délimitent d'une manière irrémédiable la distance entre le dedans et le dehors, et par conséquent contribuent à inculquer aux nouveaux venus l'illusio qui est au principe du champ.

\section{Conclusion}

Ainsi peut se dessiner à grands traits l'économie du champ juridique français. Pour être complet, il faudrait cependant aller au-delà d'une logique proprement interniste et montrer les effets du magistère des juristes sur l'ensemble de la société : on mettrait ainsi en évidence que ce capital spécifique qu'est le capital juridique constitue une condition de possibilité de domination sociale, et en particulier de leadership politique. Plus encore, on démontrerait comment, dans de multiples sphères sociales, les juristes s'efforcent d'imposer la légitimité de leur présence, de même que la nécessité d'un monopole des outils et des catégories du droit pour réguler la société et produire les normes qui la règlent ${ }^{92}$. Parmi les conditions de félicité de l'imposition d'un ordre juridique sur les différents espaces sociaux, on trouve précisément ce principe d'autonomie du champ, autonomie qui s'appuie sur une sorte de métaphysique s'élevant au-dessus des réalités sociales et des raisons pratiques propres à chaque espace social.

Pour autant, cette autonomie est toujours relative et à refaire, et elle est aujourd'hui fortement remise en cause par l'essor d'un autre mode de gouvernement des sociétés, économique et managérial. Tant et si bien que l'autonomie du champ juridique et l'existence des professions sont fortement remises en cause, et avec elles l'ensemble de la disposition juridique que cet article a tenté de déplier. Ainsi, le rapprochement du droit et de l'économie, pointé dès le début des années 1990 par Yves Dezalay à travers la figure du «marchand de droit », est aujourd'hui devenu à ce point critique que l'on assiste plutôt à une forme de fusion entre certaines fractions du champ économique et certaines fractions du champ juridique. La lutte pour l'autonomie professionnelle, menée par un certain nombre de groupes d'intérêts, et qui est finalement au cœur de l'utilité sociale de ces professions que l'on peut appeler "prudentielles », est peut-être devenue une question de survie 93 .

91. Pierre Bourdieu, «Les rites comme actes d'institution", Actes de la recherche en sciences sociales, 43, 1982, p. 58-63.

92. Sur cette question, cf. la troisième partie de Laurent WiLLEMEZ, « Des mondes ordonnés? Professionnels du droit et militants dans la diffusion de la raison juridique », op. cit.

93. Florent СНАмРY, "Pourquoi il faut défendre l'autonomie professionnelle, et comment», in Bertrand MAS, Frédéric PIERRU, Nicole SMOLSKI et al. (dir.), L’hôpital en réanimation, Bellecombe-en-Bauges : Éditions du Croquant, 2011, p. 259-268. 
Analyser les principes structurant le champ juridique a un dernier intérêt : il autorise à faire porter l'attention sur les agents du champ juridique qui tentent de le réformer, en particulier en essayant de contrarier les tendances à la fermeture ou en refusant un certain nombre de pratiques jugées corporatistes ou traditionalistes. Revendiquant des prises de position politiques et l'utilisation de leurs compétences professionnelles pour la défense de causes ${ }^{94}$, volontiers ironiques face à la disposition juridique, certains juristes, avocats, magistrats ou universitaires s'efforcent ainsi d'inventer une nouvelle manière de faire du droit et d'appartenir au champ juridique, à distance de l'orthodoxie juridique mais dans le respect d'un ensemble de règles minimales permettant de continuer à se vivre comme juristes. C'est pourquoi il est sans doute désormais nécessaire de ne plus seulement faire la sociologie des agents hétérodoxes, mais d'enquêter au cœur même du champ, chez ceux qui sont les plus pris dans le champ et qui ont le plus d'intérêt à sa préservation : l'enquête en est bien entendu plus difficile ${ }^{95}$, mais elle permettra sans doute de mieux comprendre dans le même temps les stratégies d'hétérodoxie.

On le voit, l'intérêt majeur d'utiliser les théories du champ tient peut-être d'abord dans l'analyse des limites de ce modèle que laisse voir l'enquête empirique sur les acteurs et les institutions qui le structurent: parce que l'autonomie qui en est le cœur est toujours l'objet d'une lutte, parce qu'existent diverses stratégies d'orthodoxie et d'hétérodoxie, parce que les effets de croyance et d'adhésion à l'illusio sont toujours plus nuancés que ne le présente le modèle, il faut d'abord considérer le champ comme un outil théorique, et finalement comme un idéal-type : le champ n'est pas une "chose", mais un "moyen conceptuel pour comparer et mesurer la réalité à lui » 96 . L'analyse des propriétés du champ juridique constitue bien ainsi une sorte de préalable à l'étude d'acteurs et d'institutions qui dénotent et peuvent être analysés dans la distance qu'ils présentent par rapport au concept 97 .

94. Pour une analyse exhaustive de ces questions, cf. Liora IsRAËL, L'arme du droit, Paris: Presses de Sciences Po, 2009.

95. Puisque ceux-là sont plus loin du mode de pensée sociologique leur permettant d'accepter de se soumettre à l'objectivation.

96. Max WEBER, Essais sur la théorie de la science, op. cit., p. 182.

97. Je tiens à remercier Arnaud Saint-Martin et Antoine Vauchez, ainsi que les relecteurs anonymes de la revue, pour leur lecture des différentes versions de ce texte. 


\section{口 L'auteur}

Laurent Willemez est professeur de sociologie à l'Université de Versailles-Saint-Quentinen-Yvelines et directeur du laboratoire Printemps (CNRS-UVSQ, UMR 8085). Ses recherches articulent sociologie politique, sociologie de l'engagement militant et sociologie du droit et de la justice, autour de la thématique du droit du travail. Il a travaillé plus précisément sur les avocats militants, les syndicalistes spécialisés dans l'action juridique et les conseillers prud'hommes.

Il a notamment publié :

— «Les prud'hommes et la fabrique du droit du travail : contribution à une sociologie des rôles judiciaires ", Sociologie du travail, 45 (1), 2012 ;

— Les prud'hommes. Actualité d'une justice bicentenaire (dir., avec Hélène MicHEL), Bellecombe-en-Bauges : Éditions du Croquant 2008 ;

— La justice face à ses réformateurs (dir., avec Antoine VAUCHEZ), Paris : PUF, 2007. 